\title{
Human to mosquito transmission of dengue viruses
}

\author{
Lauren B. Carrington ${ }^{1,2 *}$ and Cameron P. Simmons ${ }^{1,2,3}$ \\ 1 Dengue Group, Oxford University Clinical Research Unit, Hospital for Tropical Diseases, Ho Chi Minh City, Vietnam \\ ${ }^{2}$ Department of Microbiology and Immunology, The University of Melbourne, Melbourne, VIC, Australia \\ ${ }^{3}$ Nuffield Department of Clinical Medicine, Centre for Tropical Medicine, University of Oxford, Oxford, UK
}

Edited by:

Simona Zompi, UC Berkeley, USA

Reviewed by:

Simona Zompi, UC Berkeley, USA

Molly Ohainle, Fred Hutchinson

Cancer Research Center, USA

${ }^{*}$ Correspondence:

Lauren B. Carrington, Oxford

University Clinical Research Unit,

Hospital for Tropical Diseases, 764 Vo

Van Kiet, District 5, Ho Chi Minh City, Vietnam

e-mail: Ibcarrington@gmail.com,

Icarrington@oucru.org
The successful transmission of dengue virus from a human host to a mosquito vector requires a complex set of factors to align. It is becoming increasingly important to improve our understanding of the parameters that shape the human to mosquito component of the transmission cycle so that vaccines and therapeutic antivirals can be fully evaluated and epidemiological models refined. Here we describe these factors, and discuss the biological and environmental impacts and demographic changes that are influencing these dynamics. Specifically, we examine features of the human infection required for the mosquito to acquire the virus via natural blood feeding, as well as the biological and environmental factors that influence a mosquito's susceptibility to infection, up to the point that they are capable of transmitting the virus to a new host.

Keywords: dengue virus, transmission, Aedes aegypti, Aedes albopictus, viral titer, temperature, symptomatic vs. asymptomatic infections

\section{INTRODUCTION}

\section{THE GLOBAL DENGUE BURDEN}

The medical (1) and economic (2-7) burden of dengue is large; a recent probabilistic estimate suggested 100 million symptomatic cases occurred in 2010 (8). Human travel patterns are changing, and there is far more international traffic between dengue endemic countries and those that are usually dengue-free, albeit permissive for epidemics because of the presence of a suitable vector (9-11). This is evidenced by recent autochthonous dengue virus (DENV) transmission in Europe $(12,13)$ (local transmission subsequent to importation). The current scale of the public health problem of dengue highlights the need to better understand the underlying biological and environmental factors that result in human to mosquito transmission of DENV. A better comprehension of how these factors vary, and under what conditions, will help us to develop more effective interventions of DENV transmission.

\section{HUMAN TO MOSOUITO TRANSMISSION OF DENGUE}

Transmission of DENV from the human host to mosquitoes requires multiple biological factors to align in time and space. Under natural conditions, a susceptible mosquito can only acquire a DENV infection after it has taken a blood meal from a viremic person. When viremic blood arrives into the mosquito midgut, extracellular virus binds to undefined receptors on the cellular surface of the midgut epithelium. If the virus can successfully infect and replicate within midgut epithelium cells then new progeny virus are shed into the hemocoel (the cavity in which the hemolymph circulates, part of the open circulatory system of invertebrates), where it can subsequently disseminate and infect secondary tissues, including the salivary glands. Once sufficient virus replication has occurred in the salivary glands and upon the next probing/feeding event, the virus may be transmitted to a new host via the saliva of the infected mosquito.

\section{VECTORS OF DENV}

The primary vector of DENV is Aedes aegypti, an endophilic mosquito, preferring to live in and around homes in tropical and subtropical regions. This mosquito feeds preferentially on human blood under field conditions (14), and inhabits tropical and subtropical climates, with the geographic range spanning all continents except Antarctica. A secondary dengue vector, Aedes albopictus, is more exophilic under natural field conditions, commonly living outdoors, but still feeds almost exclusively on humans in Thailand (14), and preferentially on humans in the Indian Ocean (15). The strong preferences for human blood exhibited by these mosquitoes increase the potential for disease transmission among humans.

The expanding geographical range of DENV vectors $(16,17)$ underscores our need to examine DENV transmission dynamics in more detail. In the United States there has been a resurgence of Aedes aegypti across the South Eastern seaboard, and its presence has been noted as far north in California as South San Francisco Bay (W. K. Reisen and M. V. Armijos, UC Davis, personal communication, August 2013). Aedes aegypti is also expected to spread beyond its current range within Australia, which is presently throughout the state of Queensland, extending into the North Eastern part of Northern Territory (18). Autochthonous cases of dengue occurred in Portugal (Madeira Islands) in 2012 (13), with transmission attributed to the invasion of Aedes aegypti in the mid 2000s.

Aedes albopictus, an aggressive, nuisance day-time biter (19), is now established in numerous areas of Southern Europe (2023), with its geographic range having continuously expanded since its first observation in Albania in 1979 (24). Aedes albopictus has also become established in parts of South America and Africa that were previously free of the invasive pest (16) (and references therein). Its emergence in Australia is also a significant threat (25). This (potential and actual) range expansion of Aedes albopictus, 
particularly because it inhabits a more temperate environment than the tropical Aedes aegypti, may lead to an increased risk of DENV transmission as it brings a greater number of denguesusceptible people into contact with vectors. Photoperiod-induced diapause and non-desiccating, cold-tolerant eggs further allows Aedes albopictus to survive in cooler environments for periods of the year $(26,27)$.

Other Aedine species have been shown to be capable of transmitting DENV under experimental conditions (28-30), including Aedes polynesiensis, Aedes scutellaris, and Aedes japonicas. As discussed in Rosen et al. (28), Aedes polynesiensis has been implicated in the natural transmission of DENV, but the relative contribution of each of these mosquitoes to overall transmission has not been quantified, and is thought to be negligible (31).

\section{HUMAN FACTORS INFLUENCING TRANSMISSION}

Factors that influence the transmission of DENV from humans to mosquitoes include the following.

\section{VIRAL TITER IN HUMAN PLASMA}

The amount of virus circulating in the blood of an infected human will influence the likelihood of a mosquito becoming infected after a blood meal. Nguyen et al. (32) identified the viremia characteristics in dengue cases that led to DENV infection of blood-fed Aedes aegypti. The viremia in humans required to infect $50 \%$ of mosquitoes differed between serotypes (Figure 1). The 50\% mosquito infectious dose was $\sim 10$-fold lower for DENV-1 and DENV-2 (6.29-6.51 log10 RNA copies/ml) than for DENV-3 and DENV-4 (7.49-7.52 log10 RNA copies/ml). A dose-response relationship was observed such that with an increasing number of DENV RNA copies, there was an increased likelihood of a mosquito becoming infected, up to the point of saturation. These findings define the viremia level that interventions such as vaccines and antivirals must target for prevention or amelioration to reduce DENV transmission.

\section{DURATION OF HUMAN INFECTIOUSNESS}

Accumulated data from empirical infection studies on human subjects conducted in the first half of the twentieth century showed that humans can be infectious to mosquitoes from 1.5 days prior to the onset of symptoms to around 5 days after the commencement of symptoms (Figure 2) (33-37). In each of these studies, however, the assignment of the day or hour of the mosquito

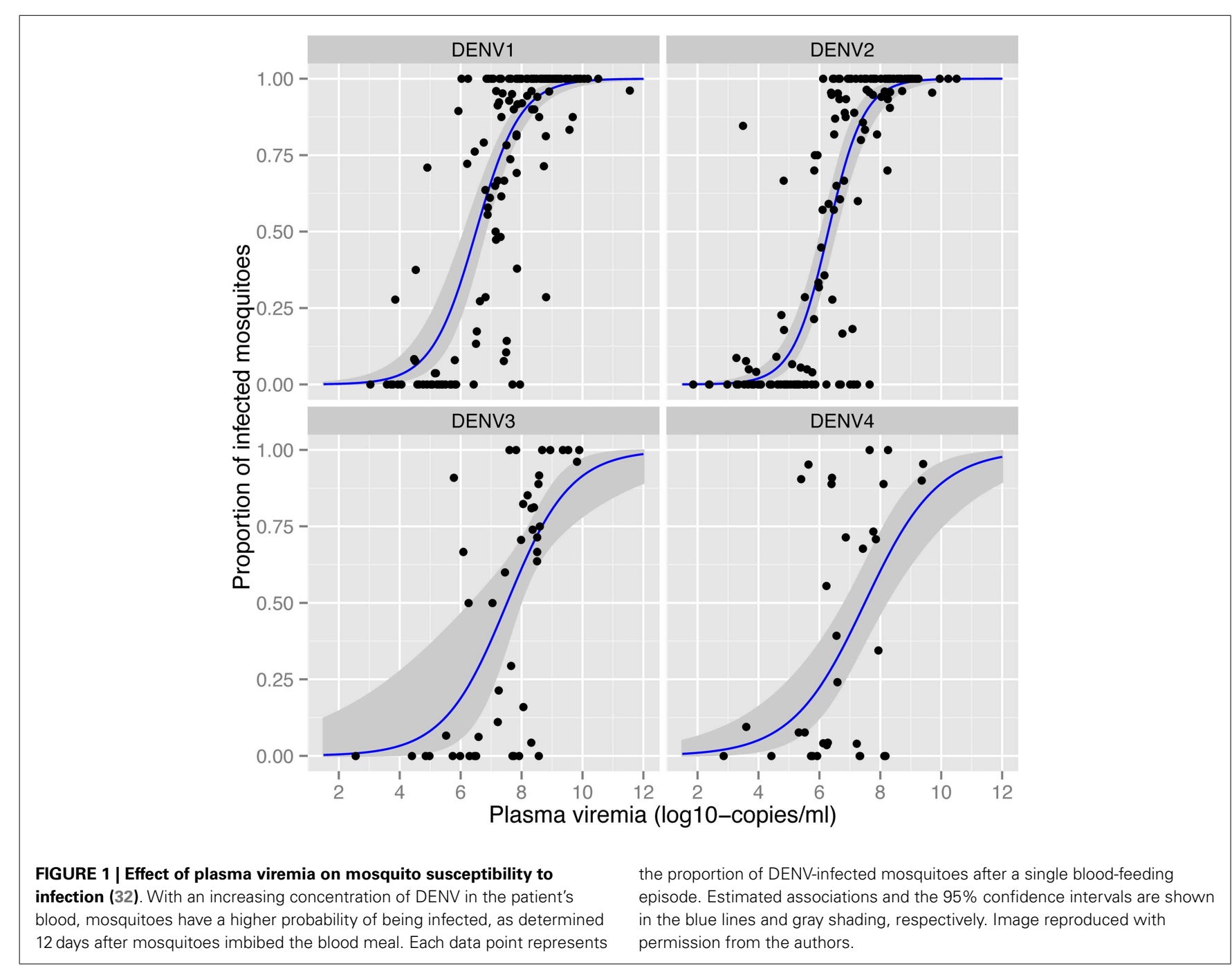


PERIOD OF INFECTIOUSNESS

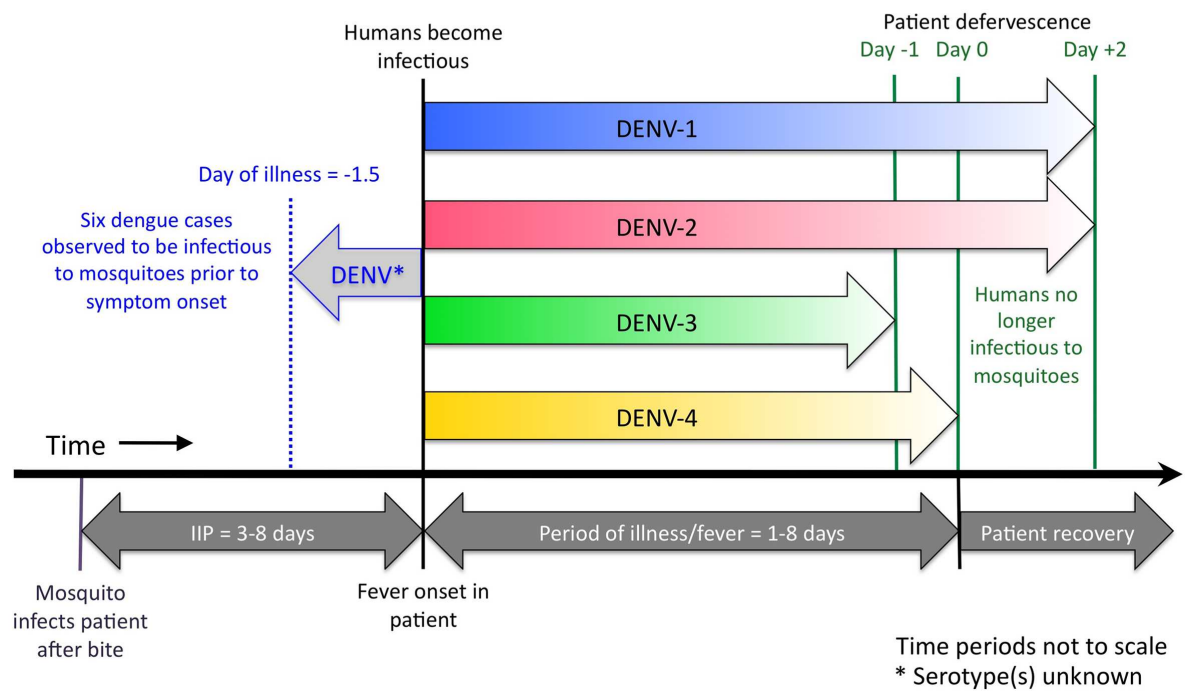

FIGURE 2 | Duration of human DENV infectiousness to Aedes aegypti mosquitoes. A person can become infectious to mosquitoes up to 1.5 days before the onset of their fever and associated symptoms, and remain infectious until the end of their febrile period, and sometimes shortly after. Indicated above the thick black line are the colored arrows showing the period of human infectiousness for each serotype, according to Nguyen et al. (32). DENV-1 (shown in blue) and DENV-2 (red) may be infectious to mosquitoes for up to 2 days after the patient becomes afebrile, however DENV-3 (green) and DENV-4 (yellow) appear to be less infectious at these later stages of illness, due to lower plasma viremia in the patient. A single study (37), found that six dengue patients were infectious to mosquitoes from 0.25 days, up to a maximum of 1.5 days, before they had any sign of symptoms (indicated by the pale gray arrow). The serotype of virus used in Siler et al.'s study is unknown. Below the black line is the relative alignment of the course of dengue illness. IIP = intrinsic incubation period. exposures was not systematic [e.g., Cleland et al. (34) exposed mosquitoes to patients on the 18 th, 22nd, 46th, 47th, 57th, 67th, and 90th hours after the onset of fever (with no apparent pattern or rationale behind the selection of these time points)], resulting in a broad range of exposure time points but with large gaps in between. In Nguyen et al.'s (32) more recent study, 208 patients who presented to the Hospital for Tropical Diseases in Ho Chi Minh City, Vietnam were enrolled in the study and randomly assigned to 2 days on which they would be exposed to naïve mosquitoes. Days of exposure ranged between day 1 and day 7 of illness. Results demonstrate that a small number of mosquitoes can still become infected with each of the four DENV serotypes up to the sixth day after illness onset. No mosquitoes became infected after feeding on patients on the seventh day after onset. Nguyen et al. (32) further demonstrated that patients with DENV-1 and DENV-2 infections can still be infectious to mosquitoes up to 2 days after defervescence, albeit this was rare. For patients infected with DENV-3 and DENV-4, viremia had declined below the required infectious dose for mosquitoes to become infected by this time. In addition, Nguyen et al. (32) demonstrated that patients with a high early viremia have a greater probability of having an extended duration of infectiousness. Intuitively, a DENV-infected person with a longer duration of viremia has a greater chance of being bitten by, and infecting, a naïve Aedes aegypti mosquito. Therefore, patients with a high early viremia generally have a greater time-window of infectiousness for mosquitoes.

\section{SYMPTOMATIC VS. ASYMPTOMATIC INFECTIONS}

Ambulatory patients with symptomatic DENV infections have viremia levels that are unquestionably likely to render them infectious to mosquitoes (32). Individuals who are asymptomatic with a DENV infection also have detectable levels of virus circulating in the blood $(38,39)$, but the question remains open as to whether or not inapparent DENV infections have high enough viremias to be infectious. Because inapparent DENV infections are common $(40,41)$, it follows that they could play a role in the maintenance of DENV in its natural transmission cycle, should their viremias be above the infectious threshold level.

\section{UNDERSTANDING THE EXTENT OF DENV TRANSMISSION THAT IS A RESULT OF ASYMPTOMATIC INFECTIONS}

While the estimated number of asymptomatic DENV infections (over 290 million cases each year) outweighs that of symptomatic infections around the world (8) the contribution of these asymptomatic infections to the continued transmission of DENV remains to be elucidated. Definitive studies to determine whether acute asymptomatic cases are able to infect susceptible mosquitoes will give insight to the contribution of asymptomatic infections to the overall transmission dynamics. These can be done in two ways. The first involves more detailed surveillance and tracing of contacts of dengue index cases than that done in current longitudinal studies [e.g., those in Thailand (40), Nicaragua (41), and Peru (42)]. Increasing the frequency of blood draws of these contacts will help to identify asymptomatic cases at the earliest possible time. At first 
observation of viremia, the individual can be exposed to susceptible mosquitoes that are then tested for infection after a suitable incubation period.

Alternatively, we can gain this same knowledge in human challenge experiments, along with vast amounts of other information, on early infection dynamics (the portion of dengue pathogenesis that is least understood because patients only present to health care professionals after symptoms have already manifested). In human challenge studies, some participants will likely develop asymptomatic infections and the question of whether these individuals are infectious to mosquitoes can be tested in a controlled setting. In addition, such studies should also take such an opportunity to study the early infection dynamics in the participants in human challenge experiments with the aim in investigating the determinants of an infection becoming symptomatic or asymptomatic.

To date, there are few studies that have even demonstrated that asymptomatic infections result in a detectable viremia. Studies in both Nicaragua and Indonesia have described persons with acute asymptomatic DENV-1 and DENV-2 infections (having successfully amplified viral RNA by RT-PCR and/or by directly isolating the virus from the blood draw) using an index-case cluster surveillance approach described above $(38,39)$. This demonstrates it is indeed possible to study asymptomatic infections within the human host, but unfortunately in both studies, DENV viremia was not quantified, and mosquitoes were not exposed to the blood of these subjects, thus it is unknown if these individuals were infectious. Duong et al. (43) reported the first and only quantification of viremias in asymptomatic cases in the literature, however, these investigators did not assess infectiousness to mosquitoes. Until empirical evidence is obtained that supports the fact that mosquitoes can become infected, and infectious, after directly feeding on asymptomatic DENV infections, one cannot ascertain the extent to which these many millions of clinically silent infections are contributing to ongoing DENV transmission, or whether they are effectively dead-end hosts.

\section{MOSQUITO SUSCEPTIBILITY TO INFECTION}

Vector competence (VC) assays of mosquito susceptibility to DENV frequently test some combination of mosquito infection, dissemination, and onward transmission of virus. One factor potentially influencing our estimates of $\mathrm{VC}$ is that many studies have used artificially derived infectious blood meals to orally infect mosquitoes. In the first half of the twentieth century, mosquitoes were routinely fed on people suffering from dengue $(33,34,36,37$, 44-48). When the weight of DENV research began to take place in non-endemic countries, a need for alternative methods arose. Since then, ordinarily, studies infect mosquitoes using artificial blood meals, consisting of a non-human blood source (often being derived from rabbit or pig), spiked with infectious virus grown in cell culture. While there are benefits of feeding mosquitoes using artificial blood meals (e.g., larger numbers of mosquitoes can be used, viral titers within the blood meal can be manipulated), employing the natural transmission mode to infect mosquitoes will help better describe the three-way human-mosquito-virus relationship in nature. Recognized factors influencing the VC of Aedes aegypti for DENV transmission are described below.

\section{RELATIVE VECTOR COMPETENCE OF AEDES AEGYPTI AND AEDES ALBOPICTUS}

Although Aedes aegypti are generally considered to be the primary vectors of DENV, Aedes albopictus have been implicated as the primary, if not the sole vector of DENV during some disease outbreaks $(49,50)$. Empirical studies show the two species do not differ in the competence to transmit DENV; both Aedes aegypti and Aedes albopictus collected from multiple sites within Cameroon showed no overall difference in their disseminated infection rate to DENV-2 (the same held true for infection with chikungunya virus also) (50). Similar results failing to identify differences in competence between the two species were reported for mosquitoes from the Florida Keys challenged with DENV-1 (51). Although both of these studies used artificial blood meals when infecting the mosquitoes and obtained similar results, the relative competence of these species after feeding on the viremic blood of a dengue case is unknown. A meta-analysis of 14 studies on the relative susceptibility of Aedes albopictus and Aedes aegypti suggests that Aedes albopictus are more susceptible to midgut infections than Aedes aegypti; however, the ability of the virus to disseminate in the latter mosquito is greater, suggesting a greater potential for transmission in nature (52).

\section{VIRUS CONCENTRATION IN THE BLOOD MEAL AND THE EXTRINSIC INCUBATION PERIOD}

Numerous studies demonstrate that the proportion of mosquitoes that become infected with DENV depends on the concentration of virus in the blood meal $(32,53)$. Bennett et al. (53) identified a positive association between viral titer of DENV-2 in the infectious blood meal and the proportion of Aedes aegypti with an infected midgut. Once infected, however, rates of dissemination in the same mosquitoes showed no such association. As described in more detail above, in more than 200 patients with naturally acquired DENV infections, Nguyen et al. (32) detected a positive correlation between mosquito infection prevalence and the titer of virus in human blood (Figure 1), consistent across all four serotypes.

Viral titer can also influence the time that it takes for a mosquito to become infectious. Watts et al. (54) demonstrated that infecting Thai Aedes aegypti with a low titer of virus resulted in an extended period (up to 25 days) before the mosquitoes were able to transmit DENV-2 to naïve rhesus monkeys, compared to when using a higher titer of virus, where it took only 12 days after incubation at the same holding temperature of $30^{\circ} \mathrm{C}$.

\section{ENVIRONMENTAL TEMPERATURE}

Environmental temperature has long been implicated in altering mosquito VC to transmit viruses. A positive correlation between mean exposure temperature and the proportion of mosquitoes that become infected with the virus exists, that is bound by upper and lower limits (54-56). The lower the temperature, the longer it takes for the virus to replicate to high enough concentrations to be transmissible (and be detectable using laboratory techniques), but at high temperatures virus replication rates are greater, and the minimum time for the mosquito to complete the extrinsic incubation is decreased. Some populations differ in these values, 
but estimates for minimum and maximum thresholds for transmission (i.e., the temperature at which a mosquito can become infectious) at constant temperatures are around $13^{\circ} \mathrm{C}$ at the lower end $(55)$, and $35^{\circ} \mathrm{C}$ at the upper end $(54,56)$ for Aedes aegypti mosquitoes (although higher temperatures are not known to have been tested). It is not known what these upper and lower limits are for Aedes albopictus.

Testing of the upper thresholds proves difficult, because after mosquitoes have been reared at such high temperatures $\left(c f .38^{\circ} \mathrm{C}\right.$ and above) the lifespan of the mosquito is reduced due to the negative effect of the high heat; their flight activity is almost negligible and they are unable to source a blood meal (57). Therefore, assessing $\mathrm{VC}$ at such high temperatures must be performed at least in semi-unnatural conditions (offering the blood meal to the mosquitoes while at a cooler temperature and then placing them back at the exposure temperatures).

Several recent VC studies investigating transmission of mosquito-borne pathogens have also shown that using natural temperature exposures (ones that fluctuate throughout the day, as a mosquito experiences in nature as opposed to constant temperatures) can alter the expected VC of a mosquito population (56, 58-60). Reaction norms for VC (and other life-history traits) as characterized under constant temperatures failed to accurately predict the competence of Aedes aegypti for DENV transmission, after exposure to the same mean temperature, but with the addition of daily temperature fluctuations. Large fluctuations in the order of $\sim 19^{\circ} \mathrm{C}$ around a low mean temperature of $20^{\circ} \mathrm{C}$ were shown to increase the number of Aedes aegypti that became infected with DENV-1, and accelerated the time that it took for dissemination to occur (by around 10 days) (56). Conversely, around a mean temperature of $26^{\circ} \mathrm{C}$, one that is commonly used for laboratory-based experiments, the same magnitude of fluctuations had the opposite effect; there were fewer mosquitoes that developed a disseminated infection, and the first time dissemination observed was extended by 4 days (60). These studies highlight that it is important to empirically test mosquitoes under conditions representative of their natural environment to accurately measure VC used for modeling purposes.

Humidity changes may also play a role in mosquito VC, but precise measurements under variable humidity regimes have not been made. It is known that desiccation under dry conditions can place mosquitoes under stress. This stress may exacerbate the inability of the mosquito to fight off a viral infection, or indeed, may negatively impact the virus, because the mosquito may utilize available cellular resources for their maintenance before the virus has the opportunity to use them. At least in Aedes albopictus, changes in humidity can enhance the effect of changes in temperature affecting mosquito fecundity (61), and it follows that this is surmised to be the same in the closely related Aedes aegypti. More in-depth studies are required to elucidate the effect of humidity on VC indices.

\section{POPULATION EFFECTS, AND INTERACTIONS BETWEEN VIRAL AND MOSOUITO GENOTYPES}

Populations of mosquitoes reportedly vary in their susceptibility to DENV infection $(53,62,63)$, which can alter the accuracy of predictions of transmission dynamics among populations. On a large geographic scale, Gubler et al. (62) demonstrated populationspecific differences in the ability of mosquitoes to become infected with each of the four DENV serotypes. Between populations, there were consistent patterns of high and low infection when exposed to each of the serotypes, suggesting that the factors controlling infection by each of the DENV serotypes is uniform and possibly conserved. Even on a relatively small scale, Bennett et al. (53) found that there was significant variation in the ability of 24 populations of mosquitoes from Mexico and USA to become infected with a DENV-2 strain.

There is also the suggestion that within a single population of mosquitoes, susceptibility to infection by different viral isolates/genotypes may vary (64-66). After challenging three isofemale lines of Aedes aegypti that were derived from Ratchaburi, Thailand, with three Thai isolates of DENV-1 virus (that were in current circulation), Lambrechts et al. (64) demonstrated that each of the Ratchaburi isofemale lines were most susceptible to infection by the viral isolate from the same city, Ratchaburi, as opposed to those from Kamphaeng Phet or Bangkok. A follow-on study identified polymorphisms at the dicer-2 locus as being associated with these phenotypic differences in mosquito VC. Further studies demonstrating that this result holds true for mosquitoes derived from other populations are needed to show the generality of the phenomenon. In any case, the differences demonstrated between mosquito populations in their susceptibility to DENV infections suggest that mathematical models of DENV transmission need to consider the nuances of specific mosquito-virus interactions in their parameterization.

\section{BLOOD-FEEDING BEHAVIOR AND PREFERENCES OF DENV MOSQUITO VECTORS}

One of the challenges standing in the way of developing targeted intervention approaches for the mosquito to human transmission cycle include a lack of understanding of mosquito behaviors, including that of host-seeking. A cornerstone of the DENV transmission cycle is the mosquito vector, and without an infected mosquito's success in seeking a suitably DENV-naïve host, transmission would cease and the virus would die. Since other bacteria and viruses manipulate the biology and behavior of their hosts to facilitate their own transmission $(67,68)$, it is plausible that DENV may do the same. Studies on the blood-feeding behavior of DENV-infected mosquitoes have examined duration of probing and feeding $(69,70)$, transmission efficiency during probing (71), and motivation and avidity to feed (72). While DENV infections may increase the duration of feeding and the likelihood of re-feeding after interruption (as tested using either mice or guinea pigs), no studies have directly investigated human host-seeking ability.

Hypothetically, if an uninfected mosquito is potentially attracted to human hosts with a high body temperature (e.g., as a result of fever), does DENV then manipulate the physiology of an infected mosquito to be more attracted to people with lower body temperature (e.g., those that are likely to be uninfected) for their subsequent meals, to increase the likelihood of transmission? Can DENV increase the frequency and/or desire to blood feed, leading to mosquito vectors feeding on multiple hosts, thereby enhancing transmission? Finally, does the virus alter the physiology of 
the human host (other than causing high fever) in ways that are detectable to a mosquito, making them more attractive? Investigating the host-feeding preferences and host-seeking ability of infected and uninfected mosquitoes can help elucidate the extent to which DENV manipulates its mosquito vectors.

\section{OTHER FACTORS INFLUENCING INFECTION}

Mosquitoes have an increased risk of infection when exposed to naturally infected dengue patients when they have a high tympanic temperature and high plasma viremia (32). With the progression of illness in a patient, IgM and IgG titers continue to increase until after viremia declines beyond a detectable limit. With this increasing day of illness and associated IgM and IgG titers, Aedes aegypti experience a decreasing risk of DENV infection (32). Increased titers of these antibodies in the blood may directly influence mosquito susceptibility, by neutralizing virus and preventing infection of the midgut.

\section{NOVEL ENTOMOLOGICAL STRATEGIES FOR REDUCING DENV TRANSMISSION IN THE FIELD}

A number of novel strategies are being developed that control mosquito populations. These include but are not limited to the use of genetic manipulations of mosquitoes, fungus, and bacteria to curb pathogen transmission. The RIDL (release of insects carrying a dominant lethal) technique releases genetically modified males into a mosquito population that carry a late-acting lethal, development gene that is transmitted to each of its progeny (73). Fungal biopesticides have also been proposed for control of mosquito transmission of pathogens (74).

Another of these strategies intends to release mosquitoes infected with the intracellular bacterium Wolbachia pipientis (75, 76). In Aedes aegypti, Wolbachia manipulate the host reproduction system to enhance its own vertical transmission between generations, but can also reduce host lifespan (77), and critically interfere with DENV replication (78). The level of virus interference in Aedes aegypti is however dependent on the bacterial strain.

Releasing mosquitoes into the wild that contain this intracellular bacterium aims to reduce the ability of mosquitoes to transmit DENV under field conditions. After the initial establishment phase of the release, this biological control strategy is self-maintained due to Wolbachia's ability to drive itself into a population of hosts, thereby increasing the benefit of this strategy by decreasing long-term maintenance costs. Additionally, the technology can be implemented relatively cheaply, meaning that countries that face a large dengue burden may see the greatest value in its implementation. There are also multiple strains of the bacteria that can be utilized, with different incompatibility phenotypes, offering the opportunity for multiple releases. Field releases of Wolbachia-infected Aedes aegypti have already occurred in Northern Australia, Vietnam, and Indonesia with the aim of suppressing DENV transmission.

One theoretical concern about this strategy is the long-term efficacy of the program due to evolutionary changes in the genomes of vector, virus, and/or bacteria. Evolution may erode the viral replication inhibition effect of the bacteria, increased virulence of the virus in humans, and decrease the life-shortening phenotype in the bacterial host, as seen in the native Drosophila host of the life-shortening Wolbachia strain (79). An objective discussion of the potential evolutionary changes in the Wolbachia vs. DENV relationship, within the human-Aedes aegypti framework, is presented by Bull and Turelli (80).

\section{SUMMARY AND RESEARCH PRIORITIES}

The successful transmission of DENV from human to mosquitoes is a complex interplay of biotic and abiotic factors. Despite this, DENV transmission occurs on a global scale and continues to be the most prevalent arbovirus infection, with an estimated 390 million infections each year (8). At this point, there are several research priorities that would benefit our understanding of human to mosquito transmission, and subsequently aid research and development into the long-term goal of finding effective tools for DENV prevention (e.g., vaccines, prophylactic or therapeutic use of antivirals, and vector control). These research priorities are:

(1) To what extent do asymptomatic infections contribute to ongoing transmission? What proportion of asymptomatic infections result in mosquitoes being capable of transmission? What is the range of viremia required for transmission to occur?

(2) Can antivirals and/or neutralizing antibodies be administered to dengue patients to reduce the potential for DENV patients to infect naïve mosquitoes? Can antibodies neutralize the virus in the mosquito before it becomes infected? How would this feasibly be administered?

(3) How will dengue vaccines modify viremia after natural exposure? Will they modify viremia to a level that prevents human to mosquito transmission?

(4) What preferences do Aedes mosquitoes show toward febrile and non-febrile hosts? Are naïve mosquitoes more attracted to febrile hosts (infected with any arbovirus)? Does DENV manipulate host-seeking behavior in infected mosquitoes?

(5) Can the likely field success of novel dengue control measures, such as Wolbachia, be predicted from laboratory studies? Which of the many and complex effects of Wolbachia on Aedes aegypti life-history traits have the greatest impact on VC?

Advances in our understanding of the DENV transmission cycle in humans and mosquitoes should support the rational development and application of interventions such as vaccines, antivirals, and novel entomological control measures.

\section{AUTHOR CONTRIBUTIONS}

Lauren B. Carrington and Cameron P. Simmons conceived and wrote the manuscript.

\section{ACKNOWLEDGMENTS}

Cameron P. Simmons is supported by the National Health and Medical Research Council, Australia (NHMRC ID: 1047282).

\section{REFERENCES}

1. Anders KL, Nguyet NM, Chau NV, Hung NT, Thuy TT, Lien le B, et al. Epidemiological factors associated with dengue shock syndrome and mortality in hospitalized dengue patients in Ho Chi Minh City, Vietnam. Am J Trop Med Hyg (2011) 84:127-34. doi:10.4269/ajtmh.2011.10-0476 
2. Coudeville L, Shepard DS, Zambrano B, Dayan G. Dengue economic burden in the Americas: estimates from dengue illness. Am J Trop Med Hyg (2009) 81:127-127.

3. Beaute J, Vong S. Cost and disease burden of dengue in Cambodia. BMC Public Health (2010) 10:521. doi:10.1186/1471-2458-10-521

4. Shepard DS, Coudeville L, Halasa YA, Zambrano B, Dayan GH. Economic impact of dengue illness in the Americans. Am J Trop Med Hyg (2011) 84:200-7. doi:10.4269/ajtmh.2011.10-0503

5. Halasa YA, Shepard DS, Zeng W. Economic cost of dengue in Puerto Rico. Am J Trop Med Hyg (2012) 86:745-52. doi:10.4269/ajtmh.2012.11-0784

6. Mogasale V, Maskery B, Lim J, Thiem VD, Tho LH, Anh DD, et al. Economic evidence for policy makers in Vietnam: dengue fever economic burden of disease and household willingness to pay for dengue vaccines. Int J Infect Dis (2012) 16:E310-310. doi:10.1016/j.ijid.2012.05.1005

7. Wettstein ZS, Fleming M, Chang AY, Copenhaver DJ, Wateska AR, Bartsch SM, et al. Total economic cost and burden of dengue in Nicaragua: 1996-2010. Am J Trop Med Hyg (2012) 87:616-22. doi:10.4269/ajtmh.2012.12-0146

8. Bhatt S, Gething PW, Brady OJ, Messina JP, Farlow AW, Moyes CL, et al. The global distribution and burden of dengue. Nature (2013) 496:504-7. doi:10.1038/nature 12060

9. Allwinn R, Hofknecht N, Doerr HW. Dengue in travellers is still underestimated. Intervirology (2008) 51:96-100. doi:10.1159/000131667

10. Schwartz E, Weld LH, Wilder-Smith A, Von Sonnenburg F, Keystone JS, Kain $\mathrm{KC}$, et al. Seasonality, annual trends, and characteristics of dengue among ill returned travelers, 1997-2006. Emerg Infect Dis (2008) 14:1081-8. doi:10.3201/ eid1407.071412

11. Napoli C, Salcuni P, Pompa MG, Declich S, Rizzo C. Estimated imported infections of chikungunya and dengue in Italy, 2008 to 2011. J Travel Med (2012) 19:294-7. doi:10.1111/j.1708-8305.2012.00640.x

12. La Ruche G, Souares Y, Armengaud A, Peloux-Petiot F, Delaunay P, Desprès $\mathrm{P}$, et al. First two autochthonous dengue virus infections in metropolitan France, September 2010. Eurosurveillance (2010) 15:19676. Available from: http://www.eurosurveillance.org/ViewArticle.aspx?ArticleId=19676

13. Alves MJ, Fernandes PL, Amaro F, Osorio H, Luz T, Parreira P, et al. Clinical presentation and laboratory findings for the first autochthonous cases of dengue fever in Madeira Island, Portugal, October 2012. Eurosurveillance (2013) 18:20398. Available from: http://www.eurosurveillance.org/View Article.aspx?ArticleId $=20398$

14. Ponlawat A, Harrington LC. Blood feeding patterns of Aedes aegypti and Aedes albopictus in Thailand. J Med Entomol (2005) 42:844-9. doi:10.1603/00222585(2005)042\\%5B0844:BFPOAA\\%5D2.0.CO;2

15. Delatte H, Desvars A, Bouetard A, Bord S, Gimonneau G, Vourc'h G, et al. Blood-feeding behavior of Aedes albopictus, a vector of chikungunya on La Reunion. Vector Borne Zoonotic Dis (2010) 10:249-58. doi:10.1089/vbz.2009. 0026

16. Benedict MQ, Levine RS, Hawley WA, Lounibos LP. Spread of the tiger: global risk of invasion by the mosquito Aedes albopictus. Vector Borne Zoonotic Dis (2007) 7:76-85. doi:10.1089/vbz.2006.0562

17. Medlock JM, Hansford KM, Schaffner F, Versteirt V, Hendrickx G, Zeller $\mathrm{H}$, et al. A review of the invasive mosquitoes in Europe: ecology, public health risks, and control options. Vector Borne Zoonotic Dis (2012) 12:435-47. doi:10.1089/vbz.2011.0814

18. Beebe NW, Cooper RD, Mottram P, Sweeney AW. Australia's dengue risk driven by human adaptation to climate change. PLoS Negl Trop Dis (2009) 3:e429. doi:10.1371/journal.pntd.0000429

19. Hawley WA. The biology of Aedes albopictus. J Am Mosq Control Assoc Suppl (1988) 4:1-40.

20. Schaffner F, Karch S. First record of Aedes albopictus (Skuse, 1894) in metropolitan France. Comptes Rendus De L Academie Des Sciences Serie IIISciences De La Vie-Life Sciences (2000) 323:373-5. doi:10.1016/S0764-4469(00) 00143-8

21. Schaffner F, Van Bortel W, Coosemans M. First record of Aedes (Stegomyia) albopictus in Belgium. J Am Mosq Control Assoc (2004) 20:201-3.

22. Delaunay P, Jeannin C, Schaffner F, Marty P. News on the presence of the tiger mosquito Aedes albopictus in metropolitan France. Arch Pediatr (2009) 16:S66-71. doi:10.1016/S0929-693X(09)75304-7

23. Schaffner F. European Centre for Disease Prevention and Control Technical Report: Development of Aedes albopictus Risk Maps. Stockholm: European Centre for Disease Control and Prevention (2009).
24. Adhami J, Reiter P. Introduction and establishment of Aedes (Stegomyia) albopictus skuse (Diptera: Culicidae) in Albania. J Am Mosq Control Assoc (1998) 14:340-3.

25. Russell RC, Williams CR, Sutherst RW, Ritchie SA. Aedes (Stegomyia) albopictus - a dengue threat for Southern Australia? Commun Dis Intell Q Rep (2005) 29:296-8.

26. Medlock JM, Avenell D, Barrass I, Leach S. Analysis of the potential for survival and seasonal activity of Aedes albopictus (Diptera: Culicidae) in the United Kingdom. J Vector Ecol (2006) 31:292-304. doi:10.3376/1081-1710(2006)31। \%5B292:AOTPFS $\$ 5D2.0.CO;2

27. Urbanski J, Mogi M, O’Donnell D, Decotiis M, Toma T, Armbruster P. Rapid adaptive evolution of photoperiodic response during invasion and range expansion across a climatic gradient. Am Nat (2012) 179:490-500. doi:10.1086/664709

28. Rosen L, Rozeboom LE, Sweet BH, Sabin AB. The transmission of dengue by Aedes polynesiensis marks. Am J Trop Med Hyg (1954) 3:878-82.

29. Moore PR, Johnson PH, Smith GA, Ritchie SA, Van Den Hurk AF. Infection and dissemination of dengue virus type 2 in Aedes aegypti, Aedes albopictus, and Aedes scutellaris from the Torres Strait, Australia. J Am Mosq Control Assoc (2007) 23:383-8. doi:10.2987/5598.1

30. Schaffner F, Vazeille M, Kaufmann C, Failloux A-B, Mathis A. Vector competence of Aedes japonicus for chikungunya and dengue viruses. Eur Mosq Bull (2011) 29:141-2.

31. Mackerras IM. Transmission of dengue fever by Aedes (Stegomyia) scutellaris walk in New Guinea. Trans R Soc Trop Med Hyg (1946) 40:295-312. doi:10.1016/0035-9203(46)90070-3

32. Nguyen MN, Duong THK, Vu TT, Nguyen THQ, Tran Nguyen BC, Vo TL, et al. Host and viral features of human dengue cases shape the population of infected and infectious Aedes aegypti mosquitoes. Proc Natl Acad Sci USA (2013) 10:9072-7. doi:10.1073/pnas.1303395110

33. Cleland JB, Bradley B. Dengue fever in Australia. Its history and clinical course, its experimental transmission by Stegomyia fasciata, and the results of inoculation and other experiments. J Hyg (1918) 16:319-418. doi:10.1017/ S0022172400006690

34. Cleland JB, Bradley B, Mcdonald W. Further experiments in the etiology of dengue fever. J Hyg (1919) 18:217-54. doi:10.1017/S0022172400007476

35. Chandler AC, Rice L. Observations on the etiology of dengue fever. Am J Trop Med (1923) 3:233-62.

36. Siler JF, Hall MW, Hitchens AP. Results obtained in the transmission of dengue fever. J Am Med Assoc (1925) 84:1163-72. doi:10.1001/jama.1925. 02660420001001

37. Siler JF, Hall MW, Hitchens AP. Dengue: Its History, Epidemiology, Mechanism of Transmission, Etiology, Clinical Manifestations, Immunity and Prevention. Manila: Bureau of Science (1926).

38. Beckett CG, Kosasih H, Faisal I, Nurhayati, Tan R, Widjaja S, et al. Early detection of dengue infections using cluster sampling around index cases. Am J Trop Med Hyg (2005) 72:777-82.

39. Reyes M, Mercado JC, Standish K, Matute JC, Ortega O, Moraga B, et al. Index cluster study of dengue virus infection in Nicaragua. Am J Trop Med Hyg (2010) 83:683-9. doi:10.4269/ajtmh.2010.10-0023

40. Yoon IK, Rothman AL, Tannitisupawong D, Srikiatkhachorn A, Jarman RG, Aldstadt J, et al. Underrecognized mildly symptomatic viremic dengue virus infections in rural Thai schools and villages. J Infect Dis (2012) 206:389-98. doi:10.1093/infdis/jis357

41. Gordon A, Kuan G, Mercado JC, Gresh L, Aviles W, Balmaseda A, et al. The Nicaraguan Pediatric Dengue Cohort Study: incidence of inapparent and symptomatic dengue virus infections, 2004-2010. PLoS Negl Trop Dis (2013) 7:e2462. doi:10.1371/journal.pntd.0002462

42. Morrison AC, Minnick SL, Rocha C, Forshey BM, Stoddard ST, Getis A, et al. Epidemiology of dengue virus in Iquitos, Peru 1999 to 2005: interepidemic and epidemic patterns of transmission. PLoS Negl Trop Dis (2010) 4:e670. doi:10.1371/journal.pntd.0000670

43. Duong V, Ly S, Try PL, Tuiskunen A, Ong S, Chroeung N, et al. Clinical and virological factors influencing the performance of a NS1 antigen-capture assay and potential use as a marker of dengue disease severity. PLoS Negl Trop Dis (2011) 5:e1244. doi:10.1371/journal.pntd.0001244

44. Carpenter DN, Sutton RL. Dengue in the Isthmian Canal Zone; including a report on the laboratory findings. J Am Med Assoc (1905) 44:214-6. doi:10.1001/jama.1905.925003000460011 
45. Ashburn PM, Craig CF. Experimental investigations regarding the etiology of dengue. J Infect Dis (1907) 4:440-75. doi:10.1093/infdis/4.3.440

46. Cleland JB, Bradley B, Mcdonald W. On the transmission of Australian dengue by the mosquito Stegomyia fasciata. Med J Aust (1916) 2:179-200.

47. Simmons JS, St. John JH, Reynolds FHK. Experimental studies of dengue. Philipp J Sci (1931) 44:1-252.

48. Sabin AB. Research on dengue during World War II. Am J Trop Med Hyg (1952) 1:30-50.

49. Metselaar D, Grainger CR, Oei KG, Reynolds DG, Pudney M, Leake CJ, et al. An outbreak of type-2 dengue fever in The Seychelles, probably transmitted by Aedes albopictus (Skuse). Bull World Health Organ (1980) 58:937-43.

50. Paupy C, Ollomo B, Kambang B, Moutailler S, Rousset D, Demanou M, et al. Comparative role of Aedes albopictus and Aedes aegypti in the emergence of dengue and chikungunya in Central Africa. Vector Borne Zoonotic Dis (2010) 10:259-66. doi:10.1089/vbz.2009.0005

51. Richards SL, Anderson SL, Alto BW. Vector competence of Aedes aegypti and Aedes albopictus (Diptera: Culicidae) for dengue virus in the Florida Keys. J Med Entomol (2012) 49:942-6. doi:10.1603/ME11293

52. Lambrechts L, Scott TW, Gubler DJ. Consequences of the expanding distribution of Aedes albopictus for dengue virus transmission. PLoS Negl Trop Dis (2010) 4:e646. doi:10.1371/journal.pntd.0000646

53. Bennett KE, Olsen KE, De Lourdes Muñoz M, Fernandez-Salas I, Farfan-Ale JA, Higgs S, et al. Variation in vector competence for dengue 2 virus among 24 collections of Aedes aegypti from Mexico and the United States. Am J Trop Med Hyg (2002) 67:85-92.

54. Watts DM, Burke DS, Harrison BA, Whitmire RE, Nisalak A. Effect of temperature on the vector efficiency of Aedes aegypti for dengue 2 virus. Am J Trop Med Hyg (1987) 36:143-52.

55. McLean DM, Miller MA, Grass PN. Dengue virus transmission by mosquitoes incubated at low temperatures. Mosq News (1975) 35:322-7.

56. Carrington LB, Armijos MV, Lambrechts L, Scott TW. Fluctuations at low mean temperatures accelerate dengue virus transmission by Aedes aegypti. PLoS Negl Trop Dis (2013) 7:e2190. doi:10.1371/journal.pntd.0002190

57. Carrington LB, Armijos MV, Lambrechts L, Barker CM, Scott TW. Effects of fluctuating daily temperatures at critical thermal extremes on Aedes aegypti lifehistory traits. PLoS One (2013) 8:e58824. doi:10.1371/journal.pone.0058824

58. Paaijmans KP, Blanford S, Bell AS, Blanford JI, Read AF, Thomas MB. Influence of climate on malaria transmission depends on daily temperature variation. Proc Natl Acad Sci USA (2010) 107:15135-9. doi:10.1073/pnas.1006422107

59. Lambrechts L, Paaijmans KP, Fansiri T, Carrington LB, Kramer LD, Thomas MB, et al. Impact of daily temperature fluctuations on dengue virus transmission by Aedes aegypti. Proc Natl Acad Sci USA (2011) 108:7460-5. doi:10.1073/pnas. 1101377108

60. Carrington LB, Seifert SN, Armijos MV, Lambrechts L, Scott TW. Reduction of Aedes aegypti vector competence for dengue virus under large temperature fluctuations. Am J Trop Med Hyg (2013) 88:689-97. doi:10.4269/ajtmh.12-0488

61. Costa EAPDA, De Mondonça Santos EM, Correira JC, De Albuquerque CMR. Impact of small variations in temperature and humidity on the reproductive activity and survival of Aedes aegypti (Diptera, Culidicae). Rev Bras Entomol (2010) 54:488-93. doi:10.1590/S0085-56262010000300021

62. Gubler D, Nalim S, Tan R, Saipan H, Sulianti Saroso J. Variation in susceptibility to oral infection with dengue viruses among geographic strains of Aedes aegypti. Am J Trop Med Hyg (1979) 28:1045-52.

63. Armstrong PM, Rico-Hesse R. Differential susceptibility of Aedes aegypti to infection by the American and Southeast Asian genotypes of dengue type 2 virus. Vector Borne Zoonotic Dis (2001) 1:159-68. doi:10.1089/153036601316977769

64. Lambrechts L, Chevillon C, Albright RG, Thaisomboonsuk B, Richardson $\mathrm{JH}$, Jarman RG, et al. Genetic specificity and potential for local adaptation between dengue viruses and mosquito vectors. BMC Evol Biol (2009) 9:160. doi:10.1186/1471-2148-9-160

65. Lambrechts L, Quillery E, Noel V, Richardson JH, Jarman RG, Scott TW, et al. Specificity of resistance to dengue virus isolates is associated with genotypes of the mosquito antiviral gene Dicer-2. Proc Biol Sci (2012) 280:20122437. doi:10.1098/rspb.2012.2437

66. Fansiri T, Fontaine A, Diancourt L, Caro V, Thaisomboonsuk B, Richardson JH, et al. Genetic mapping of specific interactions between Aedes aegypti mosquitoes and dengue viruses. PLoS Genet (2013) 9:e1003621. doi:10.1371/journal. pgen. 1003621

67. Werren JH, Baldo L, Clark ME. Wolbachia: master manipulators of invertebrate biology. Nat Rev Microbiol (2008) 6:741-51. doi:10.1038/nrmicro1969

68. Qualls WA, Day JF, Xue R-D, Bowers DF. Altered response to DEET repellent after infection of Aedes aegypti (Diptera: Culicidae) with sindbis virus. J Med Entomol (2011) 48:1226-30. doi:10.1603/ME10163

69. Putnam JL, Scott TW. Blood-feeding behaviour of dengue-2 virus infected Aedes aegypti. Am J Trop Med Hyg (1995) 52:225-7.

70. Platt KB, Linthicum KL, Myint KSA, Innit BL, Lerdthusnee K, Vaughn DW. Impact of dengue virus infection on feeding behaviour on Aedes aegypti. Am J Trop Med Hyg (1997) 57:119-25.

71. Putnam JL, Scott TW. The effect of multiple host contacts on the infectivity of dengue-2 virus-infected Aedes aegypti. J Parasitol (1995) 81:170-4. doi: $10.2307 / 3283915$

72. Maciel-de-Freitas R, Sylvestre G, Gandini M, Koella JC. The influence of dengue virus serotype-2 infection on Aedes aegypti (Diptera: Culicidae) motivation and avidity to blood feed. PLoS One (2013) 8:e65252. doi:10.1371/journal.pone. 0065252

73. Carvalho DO, Nimmo D, Naish N, Mckemey AR, Gray P, Wilke ABB, et al. Mass production of genetically modified Aedes aegypti for field releases in Brazil. J Visual Exp (2014) 83:e3579. doi:10.3791/3579

74. Thomas MB, Read AF. Can fungal biopesticides control malaria? Nat Rev Microbiol (2007) 5:377-83. doi:10.1038/nrmicrol638

75. Iturbe-Ormaetxe I, Walker GA, O'Neill SL. Wolbachia and the biological control of mosquito-borne disease. EMBO Rep (2011) 12:508-18. doi:10.1038/embor. 2011.84

76. Walker T, Johnson PH, Moreira LA, Iturbe-Ormaetxe I, Frentiu FD, Mcmeniman CJ, et al. The wMel Wolbachia strain blocks dengue and invades caged Aedes aegypti populations. Nature (2011) 476:450-5. doi:10.1038/nature10355

77. McMeniman CJ, Lane RV, Cass BN, Fong AWC, Sidhu M, Wang Y, et al. The stable introduction of a life-shortening Wolbachia infection into the mosquito Aedes aegypti. Science (2009) 323:141. doi:10.1126/science.1165326

78. Moreira LA, Iturbe-Ormaetxe I, Jeffery JAL, Lu G, Pyke AT, Hedges LM, et al. A Wolbachia symbiont in Aedes aegypti limits infection with dengue, chikungunya, and Plasmodium. Cell (2009) 139:1268-78. doi:10.1016/j.cell.2009.11.042

79. Carrington LB, Weeks AR, Hoffmann AA. The popcorn Wolbachia infection of Drosophila melanogaster: can selection alter Wolbachia longevity effects? Evolution (2009) 63:2648-57. doi:10.1111/j.1558-5646.2009.00745.x

80. Bull JJ, Turelli M. Wolbachia versus dengue: evolutionary forecasts. Evol Med Public Health (2013) 1:197-207. doi:10.1093/emph/eot018

Conflict of Interest Statement: Cameron P. Simmons is a consultant to Merck and Sanofi Pasteur for the development of dengue vaccines, and to GSK, Unither Virology, and Tibotec for the development of anti-viral therapies

Received: 17 February 2014; accepted: 03 June 2014; published online: 17 June 2014. Citation: Carrington LB and Simmons CP (2014) Human to mosquito transmission of dengue viruses. Front. Immunol. 5:290. doi: 10.3389/fimmu.2014.00290

This article was submitted to Microbial Immunology, a section of the journal Frontiers in Immunology.

Copyright $\odot 2014$ Carrington and Simmons. This is an open-access article distributed under the terms of the Creative Commons Attribution License (CC BY). The use, distribution or reproduction in other forums is permitted, provided the original author(s) or licensor are credited and that the original publication in this journal is cited, in accordance with accepted academic practice. No use, distribution or reproduction is permitted which does not comply with these terms. 